J. Lake Sci. (湖泊科学) , 2013, 25(6): 892-899

http: //www.jlakes.org. E-mail : jlakes@niglas.ac.cn

(c) 2013 by Journal of Lake Sciences

\title{
基于主成分降维的总悬浮物浓度遥感估算模型适用性分析”
}

郭宇龙, 李云梅林, 吕 恒, 王珊珊, 王永波

(南京师范大学虚拟地理环境教育部重点实验室,南京 210023)

摘 要: 总悬浮物浓度 $\left(C_{\mathrm{TSM}}\right)$ 是水质评价的重要参数. 为了提高内陆 II 类水体总悬浮物浓度估算的精度,利用主成分分 析方法对 2009 年 4 月太湖水体实测高光谱数据进行降维处理, 进而以不同数量的主成分作为变量, 分别构建总悬浮颗粒 物浓度的多元线性回归估算模型并比较这些模型的效果, 从而确定最优的主分量个数; 结合近年运行的高光谱传感器, 对模型的适用性进行评价. 结果表明: (1) 前三个主成分 $\left(P C_{1} 、 P C_{2} 、 P C_{3}\right)$ 从不同侧面涵盖了悬浮物浓度信息, 它们与 $\ln \left(C_{\mathrm{TSM}}\right)$ 的相关系数分别为 $0.728 、 0.401$ 和 0.403 ; (2) 当主成分个数为 6 时, 模型达到最优; 模型的精度高于 4 个传统经 验模型; (3) 在 $400 \sim 850 \mathrm{~nm}$ 之间, 波段数大于 45 的高光谱传感器数据都能利用主成分分析的方法构建精度较高的总悬浮 物浓度估算模型; 此外, MERIS、HJ1-HSI、Hyperion 和 CHRIS 这些常用的高光谱传感器的波段设置, 都适合于主成分建模.

关键词: 高光谱;主成分分析;降维;总悬浮物浓度;太湖

\section{Applicability analysis of the model for remotely estimating total suspended matter concen- tration based on principal component dimension reduction}

\section{GUO Yulong, LI Yunmei, LÜ Heng, WANG Shanshan \& WANG Yongbo}

(Key Laboratory of Virtual Geographic Environment of Education Ministry, Nanjing Normal University, Nanjing 210023, P. R. China)

Abstract: Total suspended matter concentration $\left(C_{\mathrm{TSM}}\right)$ is an important parameter for water quality evaluation. In this study, to improve the estimation accuracy of $C_{\mathrm{TSM}}$ in inland type II water, principal component analysis (PCA) was used to reduce the dimensions of hyperspectral data measured in Lake Taihu in April, 2009. Different multiple linear regression models of TSM were subsequently constructed using several principle components $(P C \mathrm{~s})$, and the optimal model was determined by comparing the performance of these models with each other. Finally, the applicability of the model to image data of the several current hyperspectral sensors was evaluated. The results show: (1) The first $3 P C \mathrm{~s}\left(P C_{1}, P C_{2}, P C_{3}\right)$ could explain the most of TSM variation information and the correlation coefficients between the first $3 P C \mathrm{~s}$ and $\ln \left(C_{\mathrm{TSM}}\right)$ are $0.728,0.401$ and 0.403 , respectively; (2) The optimal model could be developed when the number of $P C$ s selected to be six. The performance of the model proposed in this study is better than that of the four traditional empirical models; (3) Image data of the hyperspectral sensor that has more than 45 bands between 400 and $850 \mathrm{~nm}$ could be used to build a stable and accurate model for estimating TSM using PCA. In addition, data from frequently used sensors such as MERIS, HJ1-HSI, Hyperion and CHRIS could be also used to build this type model.

Keywords: Hyperspectral; principal component analysis; dimension reduction; total suspended matter; Lake Taihu

常规的水质监测调查速度慢、监测周期长, 难以满足对大面积水体水质监测的要求, 遥感技术可以克服 常规水质监测方法的不足. 遥感反射率是湖泊水色的综合反映, 是水体光学活性物质吸收和散射相互作用 的结果, 遥感监测水质最重要的就是建立水质参数和遥感反射率光谱之间的响应关系 ${ }^{[1]}$. 近年来, 随着高光 谱技术的迅速发展, 传感器光谱分辨率不断提高, 使得遥感反射率能够体现水色要素的细节变动, 从而提高

* 江苏高校自然科学研究重大项目 (11KJA170003)、国家自然科学基金项目 (41271343) 和江苏省 2012 年度普通高 校研究生科研创新计划项目 (CXZZ12_0397) 联合资助. 2012-11-09 收稿;2013-04-28 收修改稿. 郭宇龙, 男, 1988 年生, 博士研究生; E-mail: gyl. 18@ 163. com.

** 通信作者;E-mail:liyunmei@ njnu. edu.cn. 
水质参数反演的精度 ${ }^{[2]}$. 水体中悬浮颗粒物是重要的水色要素之一, 总悬浮物浓度 $\left(C_{\mathrm{TSM}}\right)$ 通过影响水体的 透明度、浑浊度和水色等光学性质,进而影响到水体的生态条件和初级生产力 ${ }^{[3]}$. 目前国内外许多学者通过 很多方法实现了悬浮物浓度的估算 ${ }^{[4-7]}$, 其中 (半) 经验方法具有简单快速的优势, 而且需要获取的参数少, 是最常用的估算模型构建方法. 传统的经验方法包括单波段法、波段比值法、光谱一阶微分法等 ${ }^{[8-10]}$. 然而这 些算法只能利用高光谱信息的某个或几个特征波段,建模过程中波段的选择和波段组合的方式,都包含很 多不确定因素 (例如不同时期获取的数据、最佳估算波段会有所差别), 如果都使用相同的敏感波段,会导致 最终得到的统计回归模型未必是最佳估算模型.

更多的波段会包含更多的有用信息,然而同时采用大量波段进行统计回归又存在以下问题: 高光谱数 据相邻波段相关性很高, 容易出现数据冗余, 此外, 如果将大量自变量都参与多元线性回归分析, 在增加计 算量的同时,也会使模型过于复杂, 不便使用. 偏最小二乘法 ${ }^{[11]}$ 可以较好地解决这个问题, 但对高光谱数据 来说,偏最小二乘回归很难得到一个简洁的回归方程, 不利于模型的分享,也不便应用于遥感卫星影像. 因 此本研究利用主成分分析法, 对高光谱数据进行降维处理, 以期利用较少的主分量综合反映水体光谱信息. 进而利用得到的几个互不相关的主成分对指数变换之后的悬浮物浓度进行多元回归拟合, 建立悬浮物浓度 估算模型. 最后通过实验, 确定模型的最适宜分量数以及利用该方法时高光谱数据的适宜波段数; 并对该模 型算法的遥感影像适用性进行分析, 以期提高内陆 II 类水体水色参数反演的精度, 并挖掘该模型在不同遥 感卫星影像上应用的潜力.

\section{1 材料与方法}

\section{1 研究区域}

本研究以太湖为研究区. 太湖位于长江三角洲南缘, 是我国第 3 大淡水湖泊, 流域总面积 $36500 \mathrm{~km}^{2}$, 湖 体面积 $2338 \mathrm{~km}^{2}$, 平均水深 $1.89 \mathrm{~m}^{[12]}$.

\section{2 数据的获取}

分别于 2009 年 4 月 $16-27$ 日、2011 年 5 月 20 日对太湖进行野外采样, 其中 2009 年 4 月共采集 54 个样本, 2011 年 5 月共采集 30 个 样本 (图 1). 在每个采样点获取水面遥感反射 率, 水体反射光谱的测量采用美国 ASD 公司 生产的 ASD FieldSpec Pro 便携式光谱辐射计, 其波段范围为 $350 \sim 1050 \mathrm{~nm}$. 为减少水体镜面 反射和船体自身阴影的影响, 测量时采用唐军 武等 ${ }^{[13]}$ 提出的内陆 II 类水体水面以上光谱测 量的方法. 提取遥感反射率时需要测量的数据 包括标准灰板、天空光、水体等的光谱辐亮度 信息, 每个对象都要采集 10 条以上的光谱数 据,剔除异常光谱数据,剩余数据做均值处理. 遥感反射率提取的具体方法见文献 [14]. 在 光谱测量的同时采集表层水样, 低温冷藏带回 实验室测量悬浮物的浓度. 悬浮物浓度采用常 规的干燥、烘烧、称重法 (GB/T 11901-1989)

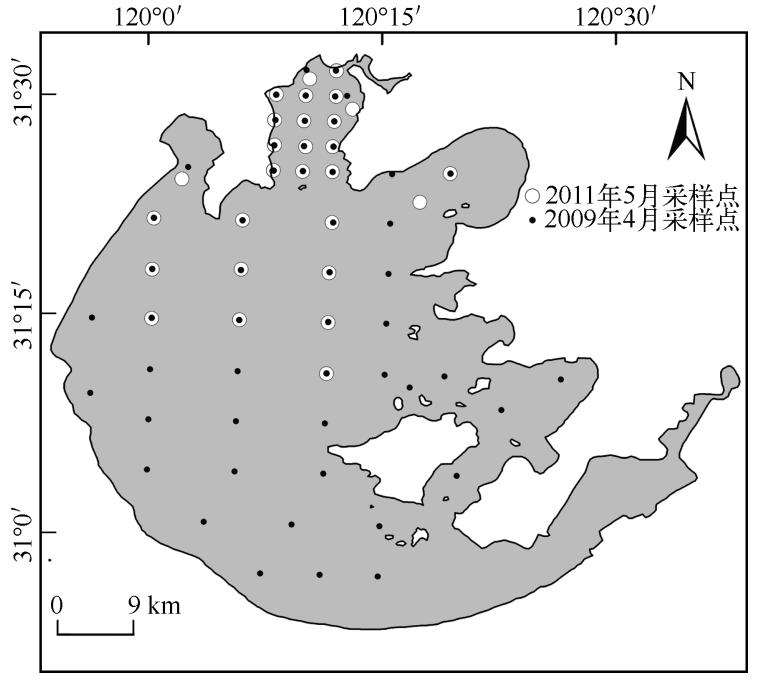

图 1 太湖采样点分布

Fig. 1 Distribution of sampling sites in Lake Taihu 测定.

\section{3 数据的处理}

为了消除不同观测时间的光谱差异, 对每条遥感反射率光谱曲线进行归一化处理(公式 (1)), 突出光谱 特征 ${ }^{[15]}$. 


$$
R_{\mathrm{rsn}}(\lambda)=\frac{R_{\mathrm{rs}}(\lambda) \cdot n}{\int_{380}^{760} R_{\mathrm{rs}}(\lambda) \mathrm{d} \lambda}
$$

式中, $R_{\mathrm{rs}}(\lambda)$ 和 $R_{\mathrm{rsn}}(\lambda)$ 分别为归一化前、后波长 $\lambda$ 处的遥感反射率, $n$ 为可见光波段参与计算的波段数, 本 研究为 381. 本研究将通过归一化前后的遥感反射率与待估算参数之间的相关性, 分析归一化前后遥感反射 率对总悬浮物浓度的敏感性.

\section{4 遥感反射率主成分分析}

主成分分析就是用少数几个主成分来描述多个指标或因素之间的联系, 以较少几个主成分反映原数据 的大部分信息的统计方法. 从数学角度来看, 是一种降维处理技术 ${ }^{[16]}$. 经分析得到的主成分用 $P C_{i}$ 表示, 如 $P C_{1}$ 代表第一主成分, $P C_{2}$ 代表第二主成分, 以此类推.

分解得到的特定主成分代表一组光谱的某个特征, 这种特征体现在该主成分的载荷系数上. 研究将通 过分析不同主成分的载荷系数,解释不同主成分在模型构建中的作用.

\section{5 基于主分量的多元线性回归建模}

高光谱数据的各个主成分之间是没有相关性的, 因此以这些主成分为自变量, 待反演参数作为因变量, 建立多元线性回归模型, 用来估算悬浮物浓度. 模型如下:

$$
y=\sum_{i=1}^{n} a_{i} \cdot P C_{i}+b
$$

式中, $y$ 为待反演的参数, 这里是 $\ln \left(C_{\mathrm{TSM}}\right)^{[17]}, n$ 为主成分个数, $a_{i}$ 为第 $i$ 个主成分的系数, $b$ 为截距项. 理论 上, 有多少个高光谱波段, 就有多少个成分分量. 因此确定模型中 $n$ 的大小也是一个关键问题. 本研究以迭 代运算的方式, 确定参与建模的主成分个数.

\section{6 模型的评价方法}

1.6.1 模型精度评价指标 研究中用于评价模型的指标均方根误差 (RMSE) 和平均相对误差 $(M A P E)$ 分别 利用式(3)、(4)计算得到:

$$
\begin{gathered}
R M S E=\sqrt{\sum_{i=1}^{n}\left(y_{i}-y_{i}^{\prime}\right)^{2} /(n-1)} \\
M A P E=\frac{1}{n} \sum_{i=1}^{n}\left|\frac{y_{i}-y_{i}^{\prime}}{y_{i}}\right|
\end{gathered}
$$

式中, $y_{i} 、 y_{i}^{\prime}$ 分别为实测悬浮物浓度和估算悬浮物浓度, $n$ 为样本数. 本研究将 2009 年 4 月实验获取的数据 分为两部分, 随机抽取其中的 36 组遥感反射率及与其对应的总悬浮物浓度作为建模数据, 用公式 (2) 来构 建总悬浮物估算模型, 剩余的 18 组数据作为验证数据, 用来验证模型的精度. 2011 年 5 月实验获取的数据 全部作为独立数据, 对模型进行独立验证. 同时, 为了对比分析, 用相同的数据建立 4 个常用的总悬浮物浓 度反演经验模型, 并与本文模型进行比较.

1.6.2 模型的影像适用性评价方法 实测高光谱数据具有 $1 \mathrm{~nm}$ 的光谱分辨率, 这是目前的高光谱影像数据 都不具备的, 因此为了评价本文模型的影像适用性, 对模型做了两类实验: 实验 1 : 从 $400 \sim 850 \mathrm{~nm}$ 共 451 个 波段中随机删除一个波段的数据(随机数由 IDL 中的 randumu 函数生成), 用剩余的波段进行主成分分析、 多元线性回归拟合建立模型, 并进行精度评价, 如此往复, 直到剩余 6 个波段为止, 作为一次实验. 重复 100 次实验, 记录每条 $R^{2}$ 曲线和 $M A P E$ 曲线,最后取均值. 以此讨论一般情况下支持主成分模型需要多少波段. 实验 2 : 通过不同传感器的波段响应函数和实测高光谱数据, 模拟一些常见传感器的光谱数据集, 针对不同 传感器模拟光谱进行模型构建, 并进行精度评价, 以此分析这些传感器对该算法的适用性.

\section{2 结果与分析}

\section{1 归一化遥感反射率的悬浮物浓度敏感性分析}

由于实测的水体反射光谱在 $850 \mathrm{~nm}$ 后迅速下降, 数据的信噪比降低, 噪声过大, 因此本研究只针对 $400 \sim$ 
$850 \mathrm{~nm}$ 范围内的光谱数据进行分析. 太湖 54 个采样点在 $400 \sim 850 \mathrm{~nm}$ 间的反射率光谱曲线具有典型的内 陆 II 类水体的光谱特征 ${ }^{[18]}$ ( 图 $2 \mathrm{~A}$ ).

归一化之后的遥感反射率在保持了原始遥感反射率特征的基础上,各个反射率曲线之间的系统差异变 小. 同时,归一化前后的相关系数曲线发生较大变化:归一化之前,相关系数曲线在 $400 \sim 850 \mathrm{~nm}$ 之间集中在 0.6 附近, 在 $400 \sim 533 \mathrm{~nm}$ 之间呈平稳下降趋势, 最小值为 0.528 , 出现在 $533 \mathrm{~nm}$ 处; $533 \sim 710 \mathrm{~nm}$ 之间平稳 上升; $710 \mathrm{~nm}$ 之后趋于平稳, 并保持在较高的水平, 最大值为 0.866 , 出现在 $730 \mathrm{~nm}$. 所有波段相关系数的方 差较小, 仅为 0.018 , 说明相关系数曲线在全部波段范围内浮动不大. 归一化之后, 相关系数曲线保持了相似 的变化趋势, 在 $400 \sim 527 \mathrm{~nm}$ 之间呈下降趋势, 最小值为 -0.898 , 出现在 $527 \mathrm{~nm}$ 处;527 650 $\mathrm{nm}$ 之间波动 上升; $650 \mathrm{~nm}$ 之后,除了在 $694 \mathrm{~nm}$ 有一个小的低值区之外, 都保持在较高的水平, 最大值为 0.911 , 出现在 $740 \mathrm{~nm}$ 处 (图 2B). 所有波段相关系数的方差为 0.399 , 表明归一化之后, 不同波段与 $\ln \left(C_{\mathrm{TSM}}\right)$ 的相关系数在 全部波段范围内波动较大.

(归一化)遥感反射率 相关系数
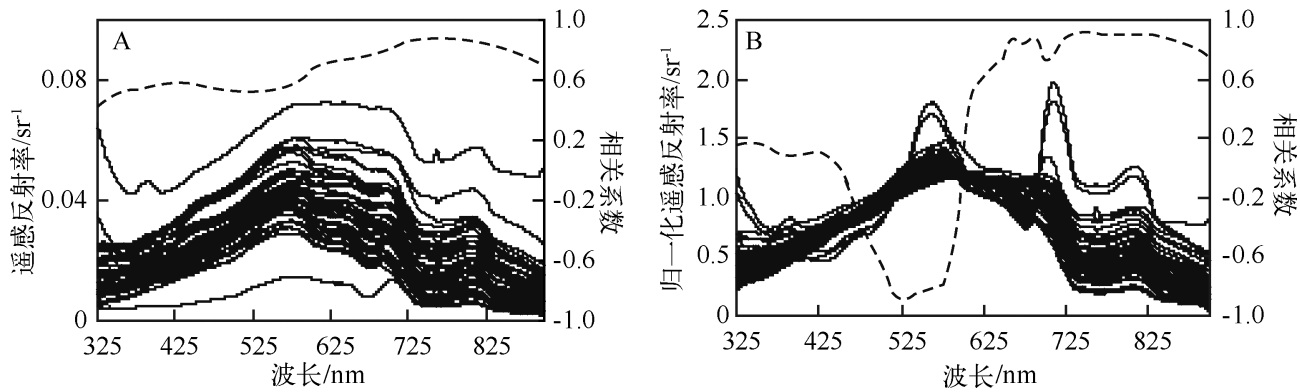

图 2 太湖遥感反射率 $(\mathrm{A})$ 、归一化遥感反射率 $(\mathrm{B})$ 及与 $\ln \left(C_{\mathrm{TSM}}\right)$ 的相关系数对比

Fig. 2 Comparison of remote sensing reflectance(A), normalized remote sensing reflectance(B) and their correlations with $\ln \left(C_{\mathrm{TSM}}\right)$ in Lake Taihu

通过对比归一化前后的相关系数曲线发 现,归一化前后敏感波段的位置变化较小, 但 数值变化明显. 最大正相关系数提高了 $5.19 \%$, 最小负相关系数降低了 $89.8 \%$, 方差 提高了 $2116.6 \%$, 可见归一化之后的遥感反射 率对总悬浮物浓度的变化更敏感, 特别是在 $510 \sim 560 \mathrm{~nm}$ 波段, 归一化之后的遥感反射率 与 $\ln \left(C_{\mathrm{TSM}}\right)$ 的相关性得到大幅度提升, 说明归 一化方法能够有效突出光谱中的总悬浮物浓 度信息.

\section{2 主分量涵盖的悬浮物浓度遥感信息分析}

首先从载荷系数曲线来看, 主成分的载荷 系数能够表明所对应的变量在主成分中所占 据的分量, 载荷系数的绝对值越大, 说明该变 量对最终主成分的影响也越大. 本研究中 $R_{\mathrm{rsn}}$ 的前三个主成分载荷曲线如图 3 所示, 其中 $P C_{1}$ 的贡献率为 $48.254 \%$, 并且载荷系数曲线 的特征与 $R_{\mathrm{rsn}}-\ln \left(C_{\mathrm{TSM}}\right)$ 相关系数曲线 (图 2B) 特征很相似, 同时都在 $700 \mathrm{~nm}$ 之后达到一

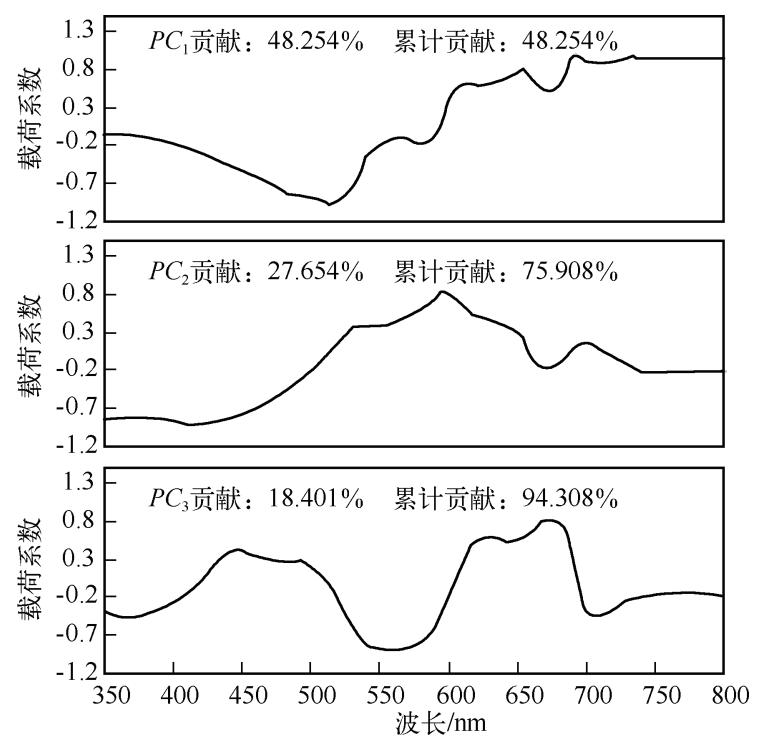

图 $3 R_{\mathrm{rsn}}$ 前三个主成分载荷、贡献率和累计贡献率

Fig. 3 Loadings, percent variance and cumulative proportion of $P C_{1}-P C_{3}$ of $R_{\mathrm{rsn}}$ 
个稳定的高值区; $P C_{2}$ 的贡献率为 $27.654 \%$, 载荷系数曲线与 $R_{\mathrm{rsn}}$ 曲线特征相似, 同时在 $700 \mathrm{~nm}$ 之后稳定在 -0.2 左右; $P C_{3}$ 的贡献率为 $18.401 \%$, 极小值出现在 $550 \mathrm{~nm}$ 附近, 对应该范围内的 $R_{\mathrm{rnn}}$ 反射峰, 之后随着波 长增加波动上升, 在 $675 \mathrm{~nm}$ 处达到峰值, 对应 $R_{\mathrm{rs}}$ 中的反射谷, 之后急剧下降, 稳定在 -0.2 附近. 从载荷系 数曲线来看, $P C_{1}$ 包含更多近红外波段的遥感反射率信息, 而近红外波段也是悬浮颗粒物的敏感波段, 由此 推断前 3 个主成分中 $P C_{1}$ 涵盖更多的悬浮物浓度信息,而 $P C_{2} 、 P C_{3}$ 则更多地体现了其余波段遥感反射率的 曲线特征.

此外, 通过对比 3 个主成分与 $\ln \left(C_{\mathrm{TSM}}\right)$ 之间的相关系数发现, $P C_{1}$ 与 $\ln \left(C_{\mathrm{TSM}}\right)$ 之间的相关系数最大, 达 到 0.728 , 验证了之前的结论; 同时, $P C_{2} 、 P C_{3}$ 与 $\ln \left(C_{\mathrm{TSM}}\right)$ 之间的相关系数分别为 0.401 和 0.403 . 可见, 虽然 每个主成分之间是不相关的, 但都体现出与 $\ln \left(C_{\mathrm{TSM}}\right)$ 之间不同程度的线性相关, 说明不同主成分可以从不 同侧面涵盖悬浮物浓度信息, 也说明悬浮物浓度的信息不仅仅包含在其敏感波段中, 也有一部分包含在非 敏感波段的反射率信息中.

至此, 前 3 个主成分的累计贡献率达到 $94.308 \%$, 理论上已经达到主成分分析的要求, 之后的主成分只 包含少量信息 (整体的 5.692\%). 然而, 水体属于弱信号体, 细微的差异就可能影响水体组分反演结果; 而细 节信号与噪声有时很难区分. 所以不能采取传统的主成分分析方法中, 简单地取累计贡献率大于 $85 \%$ 的主 成分个数进行应用的做法. 本研究中采用迭代运算的方式确定最佳主成分个数.

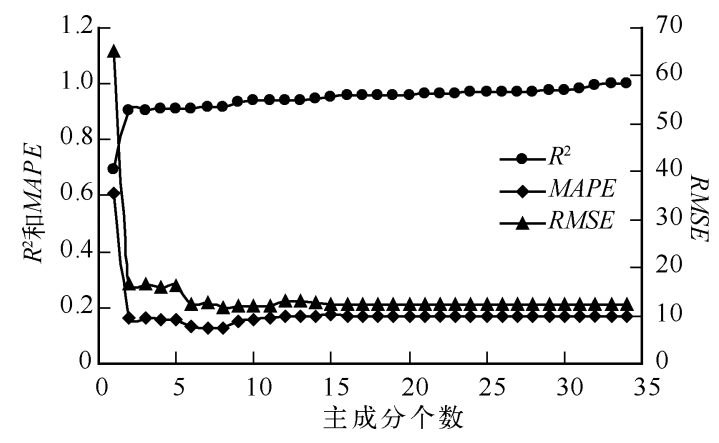

图 4 不同主成分个数下模型的 $R^{2} 、 M A P E$ 和 $R M S E$

Fig. $4 R^{2}, M A P E$ and $R M S E$ of the model using different amounts of $P C$

表 1 前 6 个主成分的特征值、贡献率和累计贡献率

Tab. 1 Eigenvalue, percent variance and cumulative proportion of $P C_{1}-P C_{6}$

\begin{tabular}{cccc}
\hline 主成分 & 特征值 & 贡献率/\% & 累计贡献率/\% \\
\hline$P C_{1}$ & 243.0131 & 53.88316 & 53.88316 \\
$P C_{2}$ & 122.3728 & 27.13365 & 81.01681 \\
$P C_{3}$ & 70.67019 & 15.66966 & 96.68648 \\
$P C_{4}$ & 11.19852 & 2.483042 & 99.16952 \\
$P C_{5}$ & 2.051273 & 0.454828 & 99.62435 \\
$P C_{6}$ & 0.560813 & 0.124349 & 99.74870 \\
\hline
\end{tabular}

\section{3 降维悬浮物浓度估算模型的构建及评价}

2.3.1 主分量个数的确定及建模 从 54 组数据中 随机抽取 36 组用作建模, 18 组用作验证, 通过不 断增加参与建模的主分量个数, 计算模型建立时 的 $R^{2}$ 和验证数据的 MAPE 及 $R M S E$, 确定对悬浮 物浓度估算最有利的主成分个数. $R^{2}$ 会随着主成 分个数增加呈不断上升的趋势 (图 4), 说明更多 的主成分能对悬浮物浓度进行更好的拟合; MAPE 和 RMSE 的变化趋势略有不同, 二者都从 2 个主 成分开始平稳下降, 到 $6 \sim 8$ 个主成分之间达到谷 值区,之后略有升高, 15 个主成分之后几乎不再变 动, 说明拟合效果最好的模型, 未必是验证估算效 果最好的模型.

从迭代结果来看, 从 2 个主成分开始, 模型的 $R^{2}$ 出现急剧上升, 与之对应的, MAPE 和 RMSE 也 都急剧下降, 之后 $R^{2}$ 趋于稳定, 而模型 $R M S E$ 和 $M A P E$ 的第二个 “突变点” 出现在 6 个主成分处. 因此取最佳主成分个数 6 个来进行多元线性回归 模型的构建和验证. 6 个主成分提取与建模的参 数如表 1 所示.

$R_{\mathrm{rsn}}$ 中的主要信息都在前 3 个主成分中得以 体现,因为从第 4 个主成分开始, 主成分的特征 值、贡献率都有急剧的变化: $P C_{4}$ 的特征值和贡献 率较 $P C_{3}$ 减小了 $84.15 \%$, 致使累计贡献率仅提高 $2.57 \%$, 之后特征值、贡献率和累计贡献率都没有

大幅度变化 (表 1). 说明 $P C_{4} \sim P C_{6}$ 更多地体现了 $R_{\mathrm{rsn}}$ 中的一些细节部分, 这些信息对 $R_{\mathrm{rss}}$ 整体的影响不大, 但却包含一些估算所需要的信息, 因此模型拟合精度 $\left(R^{2}\right)$ 才会有所提高, MAPE 和 RMSE 随之整体降低. 而 $P C_{6}$ 之后的主成分, 则更多包含 $R_{\mathrm{rsn}}$ 中的噪声信息, 不能对模型的构建产生积极的作用, 模型的 MAPE 和 $R M S E$ 也因此随之升高. 
在确定了参与建模的主成分个数之后, 得到对应的估算模型为:

$$
T S M=\exp \left(\sum_{i=1}^{n} a_{i} \cdot P C_{i}+b\right)
$$

式中, $n=6, a_{1}=0.00219, a_{2}=-0.00365, a_{3}=-0.00015, a_{4}=-0.00398, a_{5}=0.007367, a_{6}=0.124349, b=$ 3. 983785 .

建模数据集拟合的 $R^{2}$ 为 0.8441 , 验证数据集的 MAPE 为 $0.125, R M S E$ 为 12.746. MAPE 最小值仅为 0.008 , 最大值为 0.749 . 估算误差 MAPE 小于 $10 \%$ 的采样点共 11 个,占整体的 $61 \%$; MAPE 小于 $20 \%$ 的采样 点共 16 个,占整体的 $89 \%$. 说明该算法可以精确地对悬浮物浓度进行估算.

2.3.2 模型的对比分析 国内外诸多学者利用高光谱数据研发了很多悬浮物浓度的遥感估算模型,一般采 用绿光、红光波段或近红外波段来构建模型 ${ }^{[19]}$. 在此基础上,本研究构建了不同波段组合与指数变换之后的 悬浮物浓度之间的线性回归模型, 数据处理都采用相同的方法, 以避免由于处理方法不同引人新的误差. 最 终确定最大相关系数 0.823 , 对应高光谱数据 $686 \mathrm{~nm}$ 波段 $\left(R_{686}\right)$; 最小相关系数 -0.837 , 对应高光谱数据 $528 \mathrm{~nm}$ 波段 $\left(R_{528}\right)$. 于是分别以 $R_{528} 、 R_{686} 、 R_{686} / R_{528} 、 R_{686} \sim R_{528}$ 为自变量, $\ln \left(C_{\mathrm{TSM}}\right)$ 为因变量, 建立线性回归模 型, 分别记作经验模型一、二、三、四,最后得到的模型参数如表 2 所示.

无论从建模数据集拟合的 $R^{2}$, 还是验证数据集的 MAPE、 $R M S E$, 本文提出的模型都能得到较好的结果 (表 2). 与传统的 4 个经验模型相比, 本模型的拟合精度分别提高了 $9.35 \% 、 17.07 \% 、 10.60 \%$ 和 $11.32 \%$, 平均提高了 $12.01 \%$; MAPE 分别降低了 $49.51 \% 、 64.44 \% 、 53.87 \%$ 和 $53.41 \%$, 平均降低了 $56 \% ; R M S E$ 分 别降低了 $30.86 \% 、 57.66 \% 、 39.52 \%$ 和 $46.69 \%$, 平均降低了 $45.48 \%$, 可以说本模型相比于传统经验模型在 整体精度方面有显著提高.

表 2 不同模型对比

Tab. 2 Comparison of different models

\begin{tabular}{|c|c|c|c|c|c|c|c|}
\hline \multirow{2}{*}{ 模型 } & \multirow{2}{*}{ 自变量 } & \multirow{2}{*}{ 表达式 } & \multirow{2}{*}{$\frac{\text { 建模数据拟合 }}{R^{2}}$} & \multicolumn{2}{|c|}{ 验证数据 } & \multicolumn{2}{|c|}{ 独立数据 } \\
\hline & & & & MAPE & RMSE & MAPE & RMSE \\
\hline 本文模型 & $P C_{1} \sim P C_{6}$ & $T S M=\exp \left(\sum_{i=1} a_{i} \cdot P C_{i}+b\right)$ & 0.8441 & 0.1255 & 12.7463 & 0.2931 & 10.7544 \\
\hline 经验模型一 & $R_{528}$ & $T S M=\exp (-12.294 x+17.569)$ & 0.7719 & 0.2486 & 18.4357 & 0.3777 & 16.1207 \\
\hline 经验模型二 & $R_{686}$ & $T S M=\exp (6.7433 x-2.6796)$ & 0.7210 & 0.3530 & 30.1076 & 0.6082 & 24.0873 \\
\hline 经验模型三 & $R_{686} / R_{528}$ & $T S M=\exp (5.0992 x-0.6072)$ & 0.7632 & 0.2721 & 21.0755 & 0.4357 & 18.8431 \\
\hline 经验模型四 & $R_{686} \sim R_{528}$ & $T S M=\exp (4.4678 x+4.5062)$ & 0.7582 & 0.2694 & 23.9138 & 0.5463 & 21.3014 \\
\hline
\end{tabular}

由主成分模型和 4 个经验模型的实测 - 估算 散点图 (图 5) 可以看出, 验证数据集中有两个采 样点的悬浮物浓度高于 $150 \mathrm{mg} / \mathrm{L}$, 传统经验模型 在这两个点的估算中都出现了不同程度的 “偏 移” 现象, 特别是 34 号样点实测悬浮物浓度为 $237.7 \mathrm{mg} / \mathrm{L}, 4$ 种经验方法中只有 1 种能将估算误 差勉强控制在 $20 \%$ 误差线以内, 而主成分模型能 够很好地估算其浓度,使散点接近 $1: 1$ 线. 说明传 统经验模型在进行最小二乘拟合的时候受到一些 离群值的影响, 导致模型系数偏离最佳情况. 因 此, 在进行传统经验模型构建的时候, 往往要剔除 一些所谓的 “异常值”, 然而可能这些离群点中也 包含有用信息, 只是用单一波段无法将这些信息 正确地表达. 而主成分模型可以较好地修正这些 “偏移”, 说明主成分分析提供了一种更加确切的

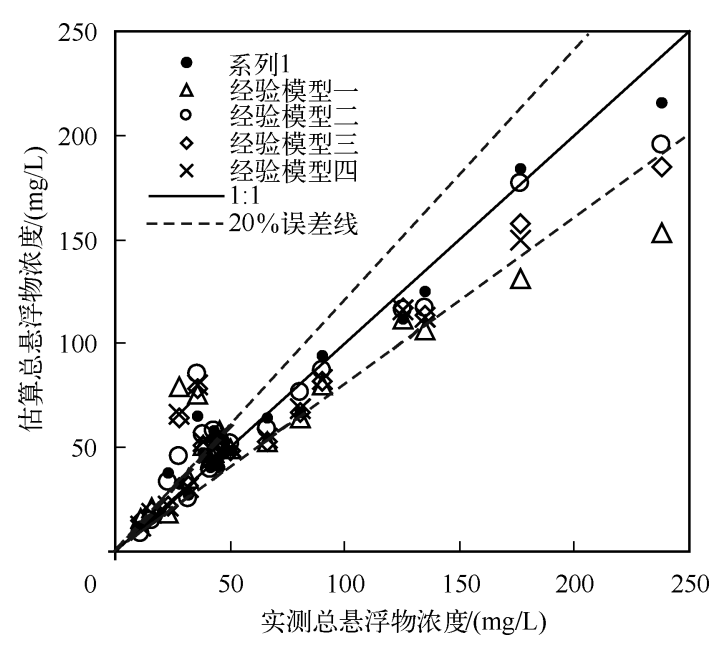

图 5 估算与实测总悬浮物浓度对比

Fig. 5 Comparison of estimated and measured TSM contents 
光谱信息提取方法,能有效地利用离群点的有用信息,并反映在最终的估算精度中.

此外, 虽然所有 5 个模型在独立数据中的 $M A P E$ 相较于验证数据都有所升高, 但本文模型仍能将 $M A P E$ 控制在 $30 \%$ 以下,优于 4 个传统的经验模型, 表明该模型更稳定,也更具实用性.

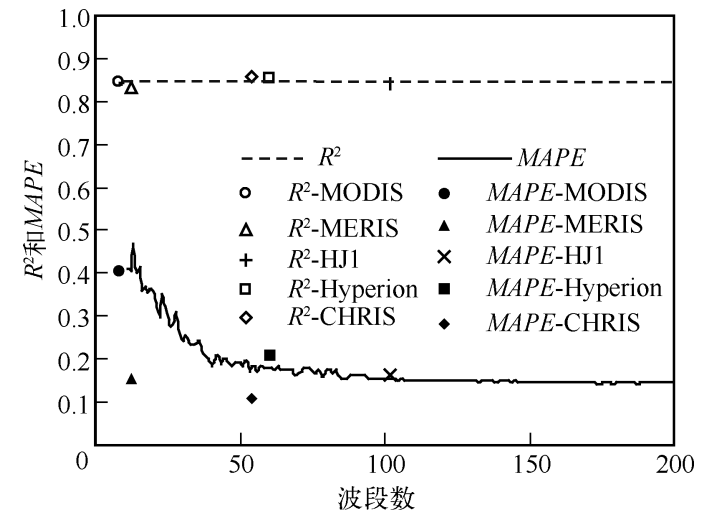

图 6 两次实验的估算模型精度

Fig. 6 Accuracy of the models in the two tests

2.3.3 模型的影像适用性评价 根据 1.6.2 中描述 的方法, 通过两组实验对本文模型的影像适用性进 行评价. 结果表明, 实验 1 中, 在波段数多于 200 时, $R^{2}$ 和 MAPE 几乎没有变化, 因此只讨论波段数 在 200 之内的情况 (图 6). 从 $R^{2}$ 曲线来看, 波段的 多少对估算模型的拟合精度影响不大, $R^{2}$ 平稳保持 在 0.85 附近. 从 $M A P E$ 曲线来看, 在波段数大于 100 的情况下, MAPE 变化同样不大,但随着波段数 量继续减少, MAPE 开始出现波动上升的趋势, 45 100 个波段之间上升幅度较小, 而波段数小于 45 之 后开始急剧升高,最后接近 0.4. 说明理论情况下, 在 $400 \sim 850 \mathrm{~nm}$ 之间,波段数量多于 45 个的传感 器数据都可以用来建立悬浮物浓度的主成分模型, 并且得到较稳定的结果, 但对于波段数量小于 45 的传感器, 模型精度和稳定性无法保证. 与 Craig 等 ${ }^{[15]}$ 用类似的方法在 I 类水体中的研究结果相比, 本研究表明需要更多的光谱来表达水体组分浓度信息, 这是因为内陆水体光学性质比较复杂, 需要更多的波段信息进行相互补充, 才能对其进行精确地表达.

实验 2 中, 共模拟了 5 种传感器的遥感反射率, 分别是 MODIS、MERIS、HJ1-HSI、Hyperion 和 CHRIS. 国 内外诸多学者对这些传感器在水色参数估算方面的应用做了大量研究, 如 Nechad 等提出一种基于多传感 器数据的浑浊水体悬浮物浓度反演算法, 并用于 MODIS、MERIS 等传感器 ${ }^{[20]}$; 杨显等基于 HJ1-HSI 数据应 用叶绿素反演的三波段并取得较高的反演精度 ${ }^{[21]}$; Liu 等基于 Hyperion 数据, 确立了珠江人海口悬浮物浓 度的波段组合模型 ${ }^{[22]}$; 李俊生等研究表明, CHRIS 数据在内陆水质监测中具有巨大潜力 ${ }^{[23-24]} .5$ 种传感器在 $400 \sim 850 \mathrm{~nm}$ 之间的波段个数分别为 $8 、 12 、 102 、 60 、 54$, 实验结果表明, 模拟数据的 $R^{2}$ 都遵循实验 1 的结果， 稳定在 0.85 附近 (图 6). 而 MAPE 体现出一些不同的特点, 其中 MODIS、Hyperion 和 HJ1-HSI 数据都在实验 1 得到的曲线附近, 三者遵循随波段数量减小, 精度降低的规律; 而 MERIS 数据和 CHRIS 数据明显分布在实 验 1 中 MAPE 均值曲线之下, 说明这两种传感器在理想状况下, 能够通过主成分模型更好地对水体悬浮物 浓度进行估算, 特别是 MERIS 数据, 虽然只有 12 个波段, 却得到仅次于 CHRIS 数据的估算精度, 说明MERIS 传感器的波段设置包含了足够的水色要素信息, 是主成分模型优秀的数据源. 最后, 除了MODIS数据以外, 参与实验的另外 4 组模拟数据都能得到较为理想的估算精度, 说明主成分建模方法在高光谱反演水体悬浮 物浓度方面具有一定的适用性.

\section{3 结论}

本文针对传统经验模型不能充分利用高光谱信息, 从而很难达到最佳拟合效果这一缺陷, 通过建立主 成分,达到利用较少分量综合反映水体信息的目的. 研究表明,水体悬浮物浓度信息不仅包含在敏感波段遥 感反射率 $\left(P C_{1}\right)$ 中,也包含在非敏感波段遥感反射率 $\left(P C_{2} \sim P C_{6}\right)$ 中.

用前 6 个主成分构建多元线性回归模型, 结果表明, 相对于 4 种传统经验模型, 主成分模型能够充分利 用高光谱数据波段信息, 受离群值影响较小, 在拟合精度和估算精度方面均有大幅度提高. 独立实验数据表 明模型具有一定实用性.

通过两类实验, 讨论了主成分模型的适用性, 结果表明, 在 $400 \sim 850 \mathrm{~nm}$ 之间波段数量大于 45 的高光谱 传感器都能用主成分模型得到高精度并且较稳定的估算模型. 并且 MERIS、HJ1-HSI、Hyperion 和 CHRIS 这 些常用的高光谱传感器的波段设置, 都适合于主成分建模, 其中 MERIS 数据虽然只有 12 个波段参与建模, 
但是其水色波段的设置,使得较少的波段也能获得较高的模型精度.

\section{4 参考文献}

[ 1 ] 周 艺,周伟奇, 王世新等. 遥感技术在内陆水体水质监测中的应用. 水科学进展, 2004,15(3):312-317.

[2] 疏小舟,尹 球,匡定波等. 内陆水体藻类叶绿素浓度与反射光谱特征的关系. 遥感学报,2000,4(1):41-45.

[ 3] 汪小钦,王钦敏,邬群勇等. 遥感在悬浮物浓度提取中的应用一一福建闽江口为例. 遥感学报, 2003,7 (1): 54-57.

[ 4 ] Gin KY, Koh ST. Spectral irradiance profiles of suspended marine clay for the estimation of suspended sediment concentration in trophical waters. International Journal of Remote Sensing, 2003, 24(16) : 3235-3245.

[ 5 ] Härmä P, Vepsäläinen J, Hannonen T et al. Detection of water quality using simulated satellite data and semi-empirical algorithms in Finland. Science of the Total Environment, 2001, 268(1) : 107-121.

[ 6 ] Ahn YH, Moon JE, Gallegos S. Development of suspended particulate matter algorithms for ocean color remote sensing. Korean Journal of Remote Sensing, 2001, 17(4) : 285-295.

[ 7 ] 刘堂友,匡定波,尹 球. 湖泊藻类叶绿素 $\mathrm{a}$ 和悬浮物浓度高光谱定量遥感模型研究. 红外与毫米波学报,2004,23 (1) $: 11-15$.

[ 8 ] 吕 恒,李新国, 江 南. 基于反射光谱和模拟 MERIS 数据的太湖悬浮物定量模型. 湖泊科学, 2005, 17 (2): 104-109.

[9] 李素菊,王学军. 巢湖水体悬浮物含量与光谱反射率的关系. 城市环境与城市生态,2003,16(6):66-68.

[10] Malthus TJ, Dekker AG. First derivative indices for the remote sensing of inland water quality using high spectral resolution reflectance. Environment International, 1995, 21(2): 221-232.

[11］张恒喜,郭基联,朱家元等. 小样本多元数据分析方法及应用.西安:西北工业大学出版社,2002.

[12] 秦伯强,胡维平,陈伟民. 太湖水环境演化过程与机理. 北京:科学出版社,2004:1-296.

[13] 唐军武,田国良,汪小勇等. 水体光谱测量与分析 I : 水面以上测量法. 遥感学报,2004,8(1):37- 45 .

[14] 唐军武. 海洋光学特性模拟与遥感模型 [学位论文]. 北京: 中国科学院遥感应用研究所, 1999:107-110.

[15] Craig SE, Jones CT, Li WKW et al. Deriving optical metrics of coastal phytoplankton biomass from ocean colour. Remote Sensing of Environment, 2012, 119(16) : 72-83.

[16] 韦玉春,王国祥,孙华芸. 使用线性回归方法构建水体叶绿素 a 浓度高光谱估算模型的一个逻辑问题. 数学的实践 与认识, $2010, \mathbf{4 0}(18): 100-110$.

[17] 刘 聪,汪 明. R 软件在主成分分析中的应用研究. 电脑知识与技术, 2011,7(13):3092-3094.

[18］施 坤,李云梅,王 桥等. 因子分析法在水质参数反演中的应用. 湖泊科学, 2010,22(3):391-399.

[19］刘忠华,李云梅,吕 恒等. 基于偏最小二乘法的巢湖悬浮物浓度反演. 湖泊科学,2011,23(3):357-365.

[20] Nechad B, Ruddick KG, Park Y. Calibration and validation of a generic multisensory algorithm for mapping of total suspended matter in turbid waters. Remote Sensing of Environment, 2010, 114(4) : 854-866.

[21] 杨 显,李云梅,王 桥等. 基于环境一号卫星高光谱遥感数据的巢湖水体叶绿素 a 浓度反演. 湖泊科学,2010,22 (4) :495-503.

[22] Liu DZ, Fu DY, Xu B et al. Estimation of total suspended matter in the Zhujiang (Pearl) River estuary from Hyperion imagery. Chinese Journal of Oceanology and Limnology, 2012, 30(1): 16-21.

[23] 李俊生,张 兵, 申 茜等. 航天成像光谱仪 CHRIS 在内陆水质监测中的应用. 遥感技术与应用, 2007,22(5): 593-597.

[24] 张 兵, 申 茜, 李俊生等. 太湖水体 3 种典型水质参数的高光谱遥感反演. 湖泊科学, 2009,21(2):182-192. 